Haben Sie Fragen zur Abrechnung oder zur wirtschaftlichen Praxisführung? Als Leser der MMW können Sie sich an unseren Experten wenden: Helmut Walbert, Facharzt für Allgemeinmedizin und Betriebswirt aus Würzburg.

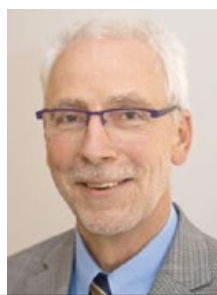

Helmut Walbert

Allgemeinarzt,

Medizinjournalist und

Betriebswirt Medizin

\title{
Und wenn der Patient die Stuhltests verschlampt?
}

B Dr. R. Z., Allgemeinärztin, Bayern: Wir haben in der Praxis immer wieder Patienten, die den Test auf okkultes Blut im Stuhl "verschlampen" oder bis zum Quartalsende nicht abgeben. Wie sollen wir das dann in der Abrechnung darstellen?

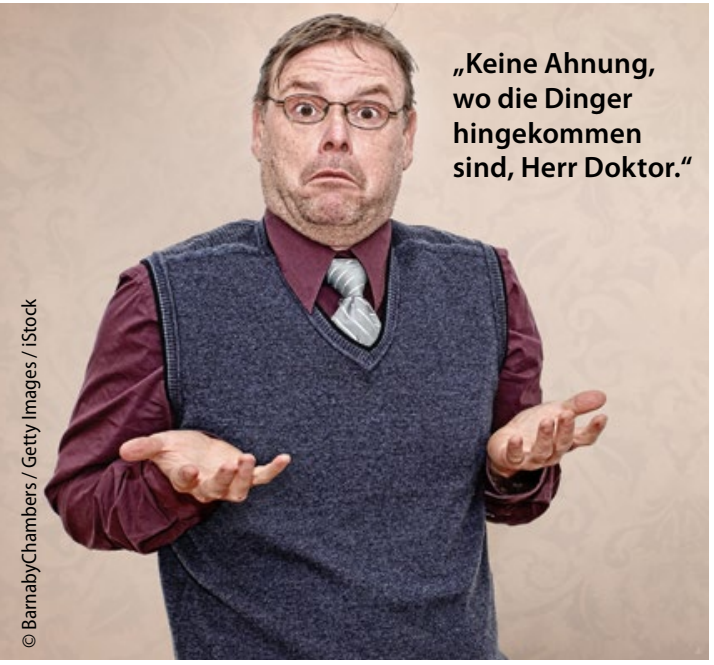

!

Antwort: Drei EBM-Ziffern spielen im Zusammenhang mit der Untersuchung auf okkultes Blut im Stuhl eine Rolle. Ich empfehle, die Nr. 01734 für die „Untersuchung auf Blut im Stuhl gemäß Krebsfrüherkennungs-Richtlinie" sofort bei der Ausgabe der Testbriefchen zu dokumentieren. So ist sichergestellt, dass die Leistung nicht verloren geht.

Die Nr. 32040 wird nur abgerechnet, wenn es sich um eine Untersuchung aus kurativem Anlass handelt. Sie ist im Behandlungsfall nicht neben der Nr. 01734 abrechenbar.

Kommen die Testbriefchen nicht zurück, wird die ursprünglich abgerechne- te Nr. gestrichen und stattdessen die $\mathrm{Nr}$ 40150 angesetzt, die Kostenpauschale für drei ausgegebene Testbriefchen, wenn die Leistungen entsprechend Nrn. 01734 oder 32040 nicht erbracht werden konnten. Dies ist auch das Vorgehen, wenn der Patient den Test als „verloren/ verschlampt " meldet oder die Tests nicht auswertbar sind.

Sie können in diesem Fall dann aber einen weiteren Versuch starten und nochmals die Nr. 01734 oder 32040 ansetzen. Es empfiehlt sich aber, das in der Abrechnung kurz zu begründen, da die Kostenpauschale in der Regel im Behandlungsfall nicht neben diesen Leistungen abgerechnet werden darf. Kommen die Tests wieder nicht zurück, müssen Sie wieder auf die Nr. 40150 umspringen.

\section{Arzneimittel-Anbieter nicht vorschnell wechseln}

$?$ H. W., Allgemeinarzt, Nordrhein: Wir bieten in unserer großen Landpraxis Hyaluron-Injektionen ins Gelenk als Selbstzahlerleistung an. Nun hat der bisherige Lieferant die Preise angehoben. Sollte ich vielleicht den Anbieter wechseln?

Antwort: Da die am Markt angebotenen Hyaluron-Präparate nicht identisch sind und ggf. nicht gleich gut vertragen werden, muss ein Anbieter- wechsel aus medizinischen Gründen wohl überlegt werden.

Sollte die Preisänderung darauf zurückgehen, dass der Anbieter einen Rabatt gekürzt hat, müssen Sie beachten, dass Rabatte beim Bezug von Arzneioder Hilfsmittel grundsätzlich in vollem Umfang an den Patienten oder die Krankenkasse weiterzugeben sind. Geschieht dies nicht, könnte das Zurückhalten der Rabatte als Abrechnungsbetrug und im Zusammenhang mit dem Antikorrup- tionsgesetz als Bestechlichkeit gewertet werden.

Ausgenommen ist die Vereinbarung eines Skontoabzuges bei Vorkasse oder umgehender Zahlung nach Rechnungseingang. Dies gilt als rechtmäßig, da hier die umgehende Liquidität für den Lieferanten zulasten der eigenen Liquidität geht. In Zeiten der Nullzinspolitik bedeutet der Skontoabzug dennoch einen nicht zu unterschätzenden Zinsgewinn für die Praxis. 